Revue d'histoire de l'Amérique française

REVUE D.HISTOIRE DE L'AMÉRIQUE FRANÇAISE

\title{
Le Commissariat canadien à Paris (1882-1928)
}

\section{Bernard Pénisson}

Volume 34, numéro 3, décembre 1980

URI : https://id.erudit.org/iderudit/303878ar

DOI : https://doi.org/10.7202/303878ar

Aller au sommaire du numéro

Éditeur(s)

Institut d'histoire de l'Amérique française

ISSN

0035-2357 (imprimé)

1492-1383 (numérique)

Découvrir la revue

Citer cet article

Pénisson, B. (1980). Le Commissariat canadien à Paris (1882-1928). Revue

d'histoire de l'Amérique française, 34(3), 357-376.

https://doi.org/10.7202/303878ar d'utilisation que vous pouvez consulter en ligne.

https://apropos.erudit.org/fr/usagers/politique-dutilisation/ 


\section{LE COMMISSARIAT CANADIEN À PARIS (1882-1928)}

BERNARD PENISSON

Lycée Sud, Le Mans

Créé en 1882, le commissariat canadien à Paris a été transformé en légation en 1928, après 46 ans d'existence. Deux titulaires seulement le dirigèrent au cours de cette période, l'un 28 ans, l'autre 17 ans, ce qui constitue sans doute un double record de longévité diplomatique dans le même poste. Le commissariat, ou mieux l'agence générale, n'était pas reconnu officiellement par les autorités françaises; car, sur le plan international, la souveraineté du Canada demeurait encore limitée par l'autorité de Londres, jusqu'à la conférence impériale de 1926. Cette institution a cependant contribué à la mise au point du système de représentation canadienne à l'extérieur ${ }^{1}$.

Nous nous proposons seulement, dans le cadre de cet article, de retracer l'évolution du poste d'après la correspondance échangée entre les premiers ministres canadiens et les commissaires. L'ambiguité des relations de la France avec le Canada est constante, car elles mettent en jeu un troisième partenaire, le RoyaumeUni. D'autre part, la province de Québec constitue un interlocuteur presque à l'égal du Canada dans les années 1880-1896, pour s'effacer ensuite ${ }^{2}$.

\section{1 - LA FONDATION DU COMMISSARIAT}

\section{A - Une initiative du Québec}

C'est la province de Québec qui a disposé la première d'un agent général à Paris, nommé par arrêté en Conseil du 28 février 1882. Pourquoi cette création? L'arrêté expliquait:

1 H. G. Skilling, Canadian Representation Abroad; from Agency to Embassy (Toronto, The Ryerson Press, 1945), XX - 359 p.

2 Sur ce sujet, Louise Beaudoin, «Origines et développement du rôle international du Gouvernement du Québec», in Le Canada et le Québec sur la scène internationale (Montréal, P.U.Q., 1977); 441-470; Armand Yon, Le Canada français vu de France (18301914) (Québec, P.U.L., 1975), 237 p.

RHAF, vol. 34, no 3, décembre 1980 
que les relations d'affaires entre la Province de Québec et le continent européen prennent tous les jours une extension croissante; que de grandes industries, des institutions financières et d'autres entreprises fondées ou alimentées par le capital français, se sont développées considérablement depuis quelque temps; que des projets importants de colonisation s'élaborent en ce moment sur le continent européen, dans le but de fonder dans cette Province divers établissements. en y envoyant des cultivateurs français, belges et flamands.

Devant cet essor des relations avec l'Europe continentale, le gouvernement du Québec estimait nécessaire, pour faciliter les contacts entre les hommes d'affaires des deux mondes, d'établir à Paris «une agence ou représentation de la Province». L'agent général agirait suivant les instructions qu'il recevrait «de temps à autre du Gouvernement de Québec». Il disposerait à Paris d'un bureau fournissant tous les renseignements officiels sur le Québec. Il devrait correspondre «autant que possible par chaque courrier océanique» avec le gouvernement de Québec. Il était plus spécialement tenu de faire «tous les six míois uin rapport général». Son traitement annuel serait de $\$ 2000$. Dans l'immédiat, il relevait «immédiatement du département du Premier Ministre». Plus tard, il dépendit principalement du ministère de la Colonisation et des Travaux publics ${ }^{4}$.

Cinq mois plus tard, le gouvernement d'Ottawa, par arrêté en Conseil du 12 juillet 1882, nommait Hector Fabre agent fédéral à Paris. Or c'était le même personnage qui représentait déjà le Québec. Une enquête, menée en 1911 par le consulat de France à Montréal sur le commissariat canadien 5 , nous apprend qu'Hector Fabre, sénateur libéral et ami du premier ministre conservateur québécois Adolphe Chapleau, avait été envoyé par ce dernier à Paris pour représenter le Québec. Fabre conservait son mandat de sénateur. Mais il ne put jouer simultanément les deux rôles et voulut abandonner son poste au Sénat. Il posait une condition: obtenir une augmentation du salaire d'agent provincial en France pour compenser la perte de son traitement sénatorial. Chapleau, devenu secrétaire d'État à Ottawa en 1882, aurait alors proposé au premier ministre fédéral sir John Macdonald, conservateur, de créer une

3 Archives Nationales du Québec (A.N.Q.), arrêté en conseil n ${ }^{\circ} 50,28$ février 1882.

4 A.N.Q., Fonds Flynn, ordre en Conseil n ${ }^{\circ} 400,2$ octobre 1896; Fonds Parent, ordre en Conseil n ${ }^{\circ} 64,14$ février 1903.

5 Archives du Ministère des Affaires étrangères (A.M.A.E.), C.P., Canada N.S. 5, $f^{\circ} 21-22,3$ avril 1911. La dépêche consulaire donne par erreur la date du 22 juillet 1882 . 
agence du gouvernement canadien à Paris. Macdonald n'en voyait pas l'utilité, d'autant plus que le Canada venait d'ouvrir à Londres, en 1879 , un haut-commissariat qui pouvait s'occuper aussi des affaires concernant le continent. Chapleau obtint alors l'appui du ministre de la Milice, Caron, dont le beau-frère, un certain de Blois, conservateur, désirait entrer à la Chambre haute. Le poste laissé vacant par le sénateur libéral Fabre favorisait ainsi le parti conservateur et Macdonald se rendit à cet argument. Ce serait la raison pour laquelle Fabre représenta le Dominion à Paris. Mais dans le premier éditorial de Paris-Canada, hebdomadaire du commissariat, Hector Fabre écrivait que c'était à cause "du désir de favoriser le rapprochement des intérêts généraux des deux pays que le gouvernement de la province de Québec a pris l'initiative de la création d'une agence canadienne à Paris et obtenu, pour cette création, le concours du gouvernement fédéral» ${ }^{6}$.

\section{$B$ - La mission fédérale de 1879 et les instructions de 1882}

Les choses ne sont peut-être pas aussi simples. La correspondance échangée entre Macdonald, Galt et Fabre montre que dès septembre 1879 le sénateur Hector Fabre se trouvait en mission à Paris, pour le compte du gouvernement fédéral, et sous le contrôle de sir Alexander Galt, le haut commissaire canadien à Londres? Fabre était chargé de mener une enquête sur le commerce du Canada avec la France et de suivre les discussions de la Chambre concernant la négociation d'un traité de commerce entre la France et les États-Unis. Il devait particulièrement informer son gouvernement sur la question de l'abaissement des taxes françaises frappant les navires canadiens. De plus, dès le 10 juillet 1880 , Fabre présentait déjà au président du Sénat, sir David Macpherson, sa démission comme sénateur de la division Lasalle ${ }^{8}$.

Le 5 juillet 1882, Macdonald avait fait à ses ministres un exposé dans lequel il signalait que Fabre, alors nommé «agent financier et commercial» du Québec à Paris, pourrait être engagé par le gouvernement fédéral pour inciter les capitalistes français à investir au Canada. Fabre devrait adresser des rapports au gouvernement, qui lui verserait aussi un traitement annuel de $\$ 2000$, plus une allocation de $\$ 500$. pour frais de bureau; il continuerait à per-

6 Paris-Canada, 11 juin 1884.

7 Archives Publiques du Canada (A.P.C.), Fonds Macdonald, M.G. 25, A, vol. 217, 3 septembre 1879, Galt à Fabre; vol. 216, 25 septembre, 15 et 29 octobre, 20 novembre 1879, Fabre à Macdonald; 15 octobre 1879, Fabre à Galt.

8 A.P.C., Fonds Macdonald, M.G. 26, A, vol. 310, f ${ }^{\circ} 140974,10$ juillet 1880. 
cevoir une somme équivalente de Québec ${ }^{9}$. Fabre reçut les instructions fédérales le 3 octobre 1882: il devait faire connaître le Canada en France et sur le reste du continent, pour orienter les capitaux et l'émigration vers le Dominion, en attirant particulièrement l'attention du public sur les ressources des forêts et des pêcheries. L'agence fédérale de Paris relevait du haut commissariat du Canada à Londres, qui faisait d'ailleurs parvenir à Fabre le montant de ses émoluments. Au Canada, le commissaire dépendait évidemment du premier ministre mais plus directement, semble-t-il, du ministère de l'Agriculture ${ }^{10}$.

\section{II - LE DÉVELOPPEMENT DU POSTE}

L'arrivée des libéraux à Ottawa entraîna quelques changements. À partir de 1902, le commissariat fut placé sous l'autorité du ministère de l'Intérieur après une tentative de rattachement à celui du Commerce. En septembre 1899, Fabre signalait au Premier ministre Laurier la possibilité de faire dépendre le commissariat du ministère de l'Intérieur, alors dirigé par Clifford Sifton:

J'ai eu la visite de Smart, le député-ministre de l'Intérieur.

(...) Il a été entendu que, sans enlever au Commissariat son caractère plus général, il se rattacherait à l'avenir plus directement au Ministère de l'Intérieur et que je correspondrai directement avec lui. Il doit faire agréer ses idées à Sifton, $(\ldots)^{11}$

Le ministère n'accorderait pour commencer que certains frais de publicité et de voyage. Mais les choses n'ont pas progressé dans cette direction, puisque le 12 juin 1901, Fabre confiait à Laurier:

Tarte m'écrit que c'est en rattachant directement le Commissariat au Ministère de l'Intérieur que les choses s'arrangeront, non pas sans doute de la façon et avec l'ampleur que vous, et lui, le souhaitiez, mais dans les seules conditions possibles pour le moment. ${ }^{12}$

Enfin, le 27 mai 1902, Fabre signalait à Laurier: «Tarte m'écrit que (...) je serai rattaché au Ministère du commerce. ${ }^{13}$

9 A.M.A.E., Canada N. S. 5, fo 13-20, 29 mars 1911.

10 Voici les adresses successives du commissariat canadien à Paris: 19 rue de Grammont, 1882-1884; 30 rue de la Rochefoucauld, 15 octobre 1884-30 juin 1885; 76 Bd Haussmann, ler juillet $1885-14$ avril 1887 ; 10 rue de Rome, 15 avril $1887-30$ juin 1911; 17/19 boulevard des Capucines, ler juillet 1911-1928.

11 A.P.C., Fonds Laurier, Fabre à Laurier, 15 septembre 1899.

12 Id., 12 juin 1901. ministre».

13 Id., 27 mai 1902. Laurier répondit le 7 juin: ...«Sifton, qui est et va rester votre 
Mais Laurier refusa, sans doute à cause de l'importance croissante de l'immigration qui dépendait de l'Intérieur.

Avec le temps, les services du commissariat avaient été étoffés. Un agent s'occupait plus spécialement de recruter des immigrants; vers 1887, ce fut Auguste Bodard, Français originaire d'Ille-et-Vilaine, qui arriva au Canada peu après 1870 et découvrit progressivement l'intérêt présenté par le Nord-Ouest pour la colonisation francophone ${ }^{14}$; il fut révoqué en 1901 pour désaccord avec le gouvernement canadien sur la politique d'immigration ${ }^{15}$. Son remplaçant, Paul Wiallard, dirigea l'agence d'immigration du ler septembre 1903 jusqu'à sa fermeture le 15 octobre $1914^{16}$. Ce bureau parisien correspondait directement avec le service général de l'immigration à Ottawa. À Paris, le Canada disposait aussi d'une agence commerciale, peut-être depuis 1896, et dont le titulaire était Anatole Poindron ${ }^{17}$; cette agence veillait au développement des relations commerciales entre le Canada et la France et dépendait du ministère du Commerce à Ottawa. Dans la pratique, ces deux agences agissaient souvent de façon indépendante du commissariat.

Poindron avait participé en septembre 1907 à la discussion du traité de commerce franco-canadien, comme secrétaire, avec Paul Wiallard, des ministres W. S. Fielding (finances) et Louis-Philippe Brodeur (marine et pêcheries). L'ambassadeur du Royaume-Uni à Paris, sir Francis Bertie, n'avait pas mené les négociations. Il avait cependant, de concert avec les ministres d'Ottawa, apposé sa signature à l'accord commercial «pour sauvegarder l'unité diplomatique de l'Empire». Le commissaire canadien se trouvait relégué quelque peu à l'écart face aux initiatives de ses agents. Mais il rencontra un allié involontaire dans le ministère français des Affaires étrangères; celui-ci, en cette période d'entente cordiale, était fort respectueux de l'unité diplomatique de l'Empire; il refusait par exemple de correspondre directement avec Poindron et adressait à l'ambassade britannique ses communications relatives au

14 Donatien Frémont, Les Français dans l'Ouest canadien (Winnipeg, les Éditions de La Liberté, 1959), 112.

is A.P.C., Id., 24 octobre et 5 novembre 1901.

16 A.P.C., Fonds Borden, Roy à Borden, 29 septembre 1914. Cette agence était située 3 rue de l'Isly. Sur Wiallard, voir R. Dandurand, Les Mémoires du sénateur Raoul Dandurand (1861-1942), édités par Marcel Hamelin (Québec, P. U. L., 1967), 144-157.

17 A.M.A.E., Canada N. S. 4, f ${ }^{\circ}$ 201-202, 18 novembre 1910. Adresses de l'agence: 101 rue de Réaumur, puis 13 rue Lafayette. Poindron avait été nommé le 29 août 1902. 
Canada ${ }^{18}$. Quant à Wiallard, sa propagande en faveur de l'immigration française au Canada lui valut de sérieux avertissements de la part des autorités françaises qui songèrent à l'expulser en $1910^{19}$. Le commissaire lui-même n'avait toujours pas le statut d'agent diplomatique et n'était pas encore accrédité auprès du gouvernement français. Fabre écrivait dès 1884 avec un sens précis des nuances: "Le Canada a un pied-à-terre à Paris; la France, un introducteur officiel au Canada. ${ }^{20}$

L'un des soucis majeurs des commissaires était donc de faire reconnaître par Ottawa leur autorité sur ces agents et de regrouper tous les bureaux canadiens dans le même immeuble parisien. Peu après l'arrivée de Wilfrid Laurier au pouvoir, en juin 1896, Fabre songeait à trouver de nouveaux locaux pour loger le commissariat qui était trop à l'étroit rue de Rome. Laurier l'approuvait et ajoutait, projetant sans doute de créer un embryon de réseau diplomatique canadien:

Non seulement il yous faudra un nouveau local, mais (...) tout le Commissariat est à reconstituer. J'ai toujours attendu l'occasion opportune. Je crois qu'elle se présentera lorsque nous aurons à faire voter l'appropriation, au printemps prochain, pour l'exposition de Paris. ${ }^{21}$

Fabre voulait en effet profiter de l'Exposition universelle de 1900, où le Canada était représenté, pour développer l'influence du commissariat canadien en France. Il voyait dans l'attribution de bureaux plus spacieux le point de départ de la réorganisation du commissariat:

Je crois qu'il faudrait profiter de l'occasion pour me confirmer mon titre de Commissaire général qui n’a pas en réalité d'existence officielle. Ce serait une reconnaissance de ma situation et une ouverture pour l'avenir. Il me resterait auprès des autorités françaises. Si je ne l'ai pas, ou l'équivalent, je me trouverai diminué aux yeux du public français. ${ }^{22}$

Ce n'est qu'en 1909 que le Département canadien des Affaires extérieures vit le jour. Le consul de France à Montréal, Joseph de Loynes, avait signalé à diverses reprises au Quai d'Orsay la tendance du gouvernement d'Ottawa à affirmer son «indépendan-

18 A.M.A.E., Canada N.S. 5, f ${ }^{\circ}$ 8-10, 23 février 1911.

19 Id., N.S. 4, f: 201-202, 18 novembre 1910.

20 Paris-Canada, 11 juin 1884.

21 A.P.C., Fonds Laurier, Laurier à Fabre, 9 novembre 1898.

22 Id., Fabre à Laurier, 15 septembre 1899. 
ce diplomatique». "C'est d'ailleurs, depuis plusieurs années déjà, le désir avoué de Sir Wilfrid Laurier.» ${ }^{23}$ Il notait que ce projet était accueilli avec enthousiasme par les libéraux mais aussi avec une ferveur marquée par les conservateurs. Le consul ne fut donc pas surpris de la création du Secrétariat d'État aux Affaires extérieures. Mais il commente avec un scepticisme mesuré cette fondation qui n'entraînera certainement pas un changement rapide du statut du commissariat canadien en France:

Le Gouvernement canadien prévoit, comme conséquence plus ou moins éloignée de la mesure actuelle, la constitution d'un personnel de Consuls, de Ministres plénipotentiaires et d'Ambassadeurs!

Il est peu probable que de si vastes projets (...) se réalisent avant bien des années. Je ne serais toutefois pas surpris que Sir Wilfrid Laurier, qui en est le véritable initiateur, réussît tout au moins à leur donner un commencement d'exécution $(\ldots)^{24}$

En décembre 1909, de Loynes signalait encore à Paris que la presse canadienne n'hésitait pas à annoncer que le Canada allait établir des consulats et même des «agences diplomatiques à l'étranger», ce qui serait, au dire des journaux, «un pas décisif vers l'autonomie complète du Canada ${ }^{25}$. Ce pays devenait vraiment une nation, mais on ne tenait pas à reconnaître le fait à Paris.

\section{III - LE RÉAMÉNAGEMENT DE 1911-1913 ET LA GUERRE}

\section{A - Une nomination délicate}

Décédé en fonction à Paris le 2 septembre 1910, Hector Fabre eut comme successeur Philippe Roy. Coïncidence curieuse, la nomination du docteur Roy aurait été reliée à la nécessité pour Wilfrid Laurier de «faire entrer au Sénat fédéral M. Forget, ancien gouverneur du Nord-Ouest» ${ }^{26}$; et pour rendre cette opération possible, le sénateur Roy devait renoncer à son siège à la Chambre haute. Avant d'être officiellement nommé, Roy posait ses conditions. Il réclamait à Laurier le titre de haut commissaire, pour obtenir dès le départ une autorité incontestée sur tous les agents

23 A.M.A.E., Canada N. S. 2, f 56-57, 24 février 1909.

24 Id., $\mathrm{f}^{\circ}$ 59-63, ler mars 1909.

25 Id., f ${ }^{\circ}$ 148-149, 7 décembre 1909. 1911.

26 Id., N. S. 5, f ${ }^{\circ} 13-20,29$ mars 1911. Roy fut nommé officiellement le ler mai 
canadiens à Paris et probablement pour se rendre indépendant du haut commissaire canadien à Londres. Il demandait à cet effet le regroupement des deux agences autour du commissariat et le contrôle effectif de tous les bureaux canadiens à Paris. Il décrivait le haut commissaire comme une sorte de "chef de bureau», ayant à son service un secrétaire général, un secrétaire pour le commerce et un secrétaire pour l'immigration; il voulait avoir droit de regard sur la nomination de ces agents. Il ne tenait pas non plus à ce que Paul Wiallard, l'agent d'émigration, se prévalût du titre de souscommissaire qu'il portait depuis le décès d'Hector Fabre dont il avait assuré l'intérim. Il exigeait enfin des appointements annuels de $\$ 12,000$, plus $\$ 3,000$ pour frais de représentation ${ }^{27}$. C'était beaucoup demander à la fois et il n'obtint de Laurier que $\$ 8,000$, complétés il est vrai par les $\$ 4,000$ versés par le gouvernement du Québec. Laurier ne lui accorda pas non plus le titre de haut commissaire, ou peut-être n'en eut-il pas le temps. Roy réussit cependant à louer des locaux plus vastes, aux 17-19 boulevard des Capucines, à partir du 1 er juillet $1911^{28}$.

La désignation du commissaire Roy permet de mieux voir la prudence du gouvernement français dans ses relations avec une "colonie» britannique. Cette réserve se manifeste à la suite d'une erreur du consul de France au Canada, Alexandre Chayet. Ce dernier venait d'être nommé à Montréal en avril 1911, mais il n'occupa ce poste que deux mois avant d'être envoyé en Australie, à Sydney, probablement à cause de sa gaffe diplomatique ${ }^{29}$. Au cours d'un entretien avec le consul, Wilfrid Laurier lui demanda s'il croyait possible d'établir des légations au Canada, malgré sa situation de dépendance britannique. Flatté d'avoir à donner un avis au premier ministre, Chayet lui cita l'exemple de l'agent et consul général de France en Égypte, vice-royauté qui relevait alors de la Turquie. Laurier parut intéressé au point de faire demander au ministère des Affaires étrangères un exposé sur la situation juridique du représentant français au Caire ${ }^{30}$. Pendant la même entrevue, interprétant trop largement ses instructions ${ }^{31}$, Chayet fit

27 A.P.C., Fonds Laurier, Roy à Laurier, 14 novembre 1910.

28 Il ne désirait d'ailleurs pas en ouvrir les portes «aux émigrants en blouse bleue et aux paysans en sabots de bois», A.P.C., Fonds Borden, Roy à Borden, 17 octobre 1911.

29 Pierre Savard, Le consulat général de France à Québec et à Montréal de 1859 à 1914 (Québec, P.U.L., 1970), 22.

30 A.M.A.E., C.P., Canada N. S. 5, f ${ }^{\circ} 23$, ler mai 1911.

31 Le ministère voyait avec déplaisir la nomination de Roy à cause de ses activités financières. Il demandait au consul «d'intervenir tout officieusement à Ottawa» pour faire 
observer à Laurier que le Canada n'avait pas demandé l'assentiment du gouvernement de la République pour la nomination du sénateur Roy. Le premier ministre lui répondit alors:

J'attendais, pour soumettre la nomination de Mr Roy à la signature du Gouv[erneu]r G[énér]al, que vous ayez reçu de votre Gouv[ernemen]t, (...) son assentiment à cette nomination. ${ }^{32}$

Le consul s'attira en retour cette note télégraphique du ministre des Affaires étrangères Jean Cruppi, visiblement gêné par l'exploitation que le gouvernement canadien pourrait faire de l'attitude française auprès de l'Angleterre:

Les fonctions de Commissaire G[énér]al à Paris n'ayant aucun caractère diplomatique ni consulaire, nous n'avons point d'avis officiel à donner sur la nomination de M. Roy. Il convient que vous le fassiez observer à Sir Wilfrid Laurier, afin qu'il ne puisse pas faire état, notamment auprès du G[ouvernemen]t Britannique, d'un assentiment que nous aurions donné, et par suite d'une reconnaissance of ficielle par nous de l'institution à Paris d'un commissariat général canadien. ${ }^{33}$

Le ministre fit également rédiger une note à l'usage de la direction des affaires politiques et commerciales de l'Amérique. Il y rappelait que son département évitait d'encourager ou de contrarier «toute tendance du Canada à accroître son autonomie ou à s'assurer une représentation extérieure indépendante de l'Angleterre». Il ajoutait:

C'est une question qui regarde exclusivement la Grande-Bretagne et sa colonie et il n'y a qu'inconvénients à s'aventurer ou à se laisser attirer sur ce terrain. ${ }^{34}$

Ce texte appelle deux remarques. Le Canada y est qualifié de «colonie», ce qui montre l'interprétation restrictive faite par le ministère de l'«autonomie» reconnue au Canada. D'autre part, il insinue que le consul de France s'est laissé attirer par Laurier sur un terrain piégé. Cette manoeuvre risquait de froisser le partenaire

connaître ce déplaisir. Mais il ajoutait: «Cette démarche doit être d'autant plus discrète et moins directe que nous sommes peu fixés sur la portée qu'attribue le gouvernement canadien aux fonctions de son Commissaire général à Paris.»Id., f ${ }^{\circ} 8-10,23$ février 1911 . En s'adressant à Laurier en personne, le consul avait été un peu trop direct.

$32 I d ., \mathrm{f}^{\circ} 24,2$ mai 1911.

33 Id., $\mathrm{f}^{\circ} 25,4$ mai 1911.

$34 I d$., $\mathrm{f}^{\circ} 28$, s. d. (note insérée entre les documents du 6 et du 12 mai 1911). 
de l'Entente cordiale. Pour éviter le retour de tout faux pas de ce genre, une nouvelle note fut rédigée en janvier 1912 à l'intention du directeur de la section Amérique. Elle précisait la discrétion à observer dans les relations avec l'agent général entretenu par le Canada à Paris:

En vue d'éviter tout froissement au gouvernement britannique nous avons toujours refusé au dit agent un caractère officiel; il est seulement considéré comme un délégué officieux du Canada. ${ }^{35}$

\section{B - Le gouvernement conservateur et les instructions de Foster}

Le 21 septembre 1911, moins de cinq mois après la nomination de Philippe Roy, les élections générales voyaient la victoire du parti conservateur et de son chef Robert Borden. Dès lors, l'orientation imprimée par Laurier à l'agence générale de Paris fut infléchie dans un sens pratique, et strictement limitée. L'ordre en Conseil du 23 octobre 1911 transférait le commissariat sous l'autorité du ministère du Commerce dont il avait failíi dépendre en $\hat{1} \hat{9} \hat{\mathbf{L}}$. George Foster, le ministre du Commerce du nouveau gouvernement, envoya ses instructions à Philippe Roy le 29 avril $1912^{36}$.

Roy ne tenait pas en effet à rester dans la position inconfortable de son prédécesseur qui semblait parfois ignorer de qui il dépendait et recevait ses instructions de différents ministères. Il avait écrit fermement en ce sens à Borden en décembre $1911^{37}$, qualifiant cette situation d' «intolérable». Il n'admettait pas qu'un fonctionnaire d'Ottawa, comme le superintendant de l'immigration W. D. Scott, puisse lui dicter sa conduite en lui imposant d'accepter des employés qu'il n'avait pas choisis. Il se méfiait particulièrement de Wiallard qui avait tenté de recueillir la succession de Fabre au commissariat; il ne voulait pas voir l'agent d'immigration travailler dans le même immeuble que lui sans relever de son autorité «d'aucune manière» comme le précisait $\mathrm{Scott}^{38}$, ce qui aurait diminué son propre statut de commissaire aux yeux des autorités françaises.

Borden demanda le temps de consulter Foster et ce fut ce dernier qui précisa les intentions du gouvernement. Il rappelait à Roy

35 Id., Canada N. S. 20, $\mathrm{f}^{\circ} 158,31$ janvier 1912.

36 A.P.C., Fonds Borden, Foster à Roy, 29 avril 1912.

37 Id., Roy à Borden, 22 décembre 1911.

38 Id., Scott à Wiallard, f ${ }^{\circ} 99618$. 
que le commissariat était principalement une agence commerciale, destinée à étudier le marché français pour favoriser l'exportation de produits canadiens, à renseigner les Français intéressés aux ressources du Canada et à mettre en contact les producteurs canadiens et les acheteurs étrangers. Dans une moindre mesure, l'agence pouvait favoriser l'entrée de produits étrangers au Canada. Le commissaire ne devait en aucun cas faire lui-même partie de sociétés financières ou les favoriser par son action, car il engageait le gouvernement fédéral. Il ne devait pas non plus, pour les mêmes raisons, avoir de liens avec des compagnies appelant le public à investir au Canada. Or Philippe Roy était président de la Jasper's Company d'Edmonton, société immobilière de spéculation sur les terrains de l'Alberta, et administrateur de la Caisse hypothécaire canadienne, fondée avec des capitaux français, dont le siège social était à Winnipeg ${ }^{39}$. Il lui fallait donc renoncer à ses intérêts privés.

Foster précisait surtout que le poste de Paris n'était en aucun cas une ambassade. Il devait rester «uniquement et strictement une agence commerciale et d'affaires, là comme ailleurs». En conséquence, le salaire du commissaire était trop élevé et serait ramené à $\$ 4,000$ par an. Le gouvernement conservateur mettait donc brusquement fin à la discrète transformation des agences canadiennes en consulats amorcée par Laurier.

Foster demandait aussi au commissaire général, qui représentait à Paris le gouvernement du Québec, de mettre un terme à cette activité. Il se disait persuadé:

qu'il n'est pas sage que ces deux fonctions soient remplies par la même personne, compte-tenu des intérêts des deux gouvernements. Des frictions et des confusions pourraient en résulter, et le meilleur moyen d'éviter des difficultés de cette sorte est de maintenir les représentations absolument séparées et distinctes. ${ }^{40}$

Pourtant, de 1882 à 1911 , aucun incident ne semble avoir surgi à propos du Québec à Paris. Il est vrai qu'au cours de cette période, les conservateurs puis les libéraux se trouvèrent au pouvoir, à peu près en même temps, à la fois à Ottawa et à Québec, alors qu'en 1911 les conservateurs dirigeaient le fédéral et les libéraux gouvernaient la «belle province».

39 A.M.A.E., Canada N. S. 5, f ${ }^{\circ}$ 13-20, 29 mars 1911.

40 A.P.C., Fonds Borden, Foster à Roy, 29 avril 1912. 


\section{C - La fin de la représentation du Québec}

Dans sa réponse à Ottawa ${ }^{41}$, Roy insistait sur les frais de représentation du commissaire canadien à Paris, car il y avait des obligations variées et onéreuses attachées à cette fonction. De plus, Roy avait une famille nombreuse, cinq enfants en 1912, alors que son prédécesseur n'avait qu'un fils, d'ailleurs décédé en 1902. En conséquence, il n'était pas disposé à renoncer à ses responsabilités d'affaires.

Il exposa ses vues au premier ministre Borden lors du voyage de ce dernier en Europe, en juillet-août 1912 ${ }^{42}$. Il mit l'accent sur la nécessité de placer directement le commissariat général sous l'autorité du premier ministre, pour augmenter le prestige du poste $^{43}$. En ce qui concernait l'ambassadeur britannique, Roy continuerait à faire preuve de la plus entière discrétion, et aucun de ses actes ne pourrait être interprété comme une usurpation des prérogatives du représentant de Sa Majesté.

Le commissaire ne voulait pas non plus, toujours pour des raisons financières, renoncer à représenter le Québec. Il avait demandé $\$ 12,000$ au gouvernement Laurier et obtenu $\$ 8,000$, à condition de toucher encore $\$ 4,000$ comme agent de la province de Québec. Il rappelait qu'aucune divergence d'intérêt ne s'était manifestée entre Québec et Ottawa à propos de la France en trente ans et que ce succès disposait le gouvernement du Québec à ouvrir une agence provinciale à Londres ${ }^{44}$.

Le gouvernement fédéral donna satisfaction à Philippe Roy pour le salaire, qui passait de $\$ 8,000$ à $\$ 12,000$. Mais le commissaire ne devait plus représenter à la fois Québec et Ottawa à Paris. Le premier ministre fournissait la raison suivante, qui avait déjà été formulée par Foster:

il n'est pas désirable que le représentant du Gouvernement Fédéral (...) soit aussi celui de l'une des neuf Provinces du Canada ${ }^{45}$ considérée comme distincte des autres. Une telle situation est sujette à mener au malentendu et à la confusion. ${ }^{46}$

41 Id., Roy à Foster, 28 mai 1912.

42 Id., Roy à Borden, 28 août 1912 .

43 Il revint encore sur ce point un mois plus tard, id., 25 septembre 1912.

44 Id., Roy à Foster, 28 mai 1912.

45 Terre-Neuve n'entra dans la Confédération qu'en 1949.

46 Id., Borden à Roy, 18 octobre 1912 . 
Première réaction d'Ottawa contre une esquisse de statut international du Québec? "L'attitude de Borden est en tout cas celle d'un précurseur de monsieur Pierre Elliot Trudeau qui cherchera toujours à contenir, dans des limites strictement définies, la vocation externe du Québec», écrit Louise Beaudoin ${ }^{47}$. Il s'agirait plutôt d'une simplification des tâches du commissaire et d'un ajustement à la situation politique fédérale-provinciale. La politique de séparation de l'Église et de l'État avait d'ailleurs dressé une partie de l'opinion ou des sentiments du Québec contre la France «officielle», ce qui explique en partie le peu d'empressement de la province à se faire représenter à Paris en $1913^{48}$. Le 9 janvier 1913, Philippe Roy offrit donc sa démission au premier ministre sir Lomer Gouin, comme agent général de la province de Québec à Paris $^{49}$.

Depuis l'installation du commissariat boulevard des Capucines, en juillet 1911, l'agence commerciale y avait été transférée ${ }^{50}$. Puis, le 15 octobre 1914, Ottawa fit fermer l'agence d'immigration à Paris ${ }^{51}$. Roy était parvenu à ses fins en un temps relativement bref. Le commissaire général avait maintenant sous son contrôle direct tous les services officiels canadiens en France. Quant à lui, il relevait désormais directement du premier ministre depuis le ler janvier $1913^{52}$. Mais il lui manquait encore l'essentiel: être accrédité auprès du gouvernement de la République. Et pour obtenir cette reconnaissance, il fallait que le Canada fût indépendant. La Grande Guerre devait accélérer cette évolution.

\section{D - La guerre}

La première guerre mondiale permit au commissariat de développer son rôle en France dans des conditions politiques très favorables. Les Canadiens, les Britanniques et les Français étaient devenus des alliés et des frères d'armes. Roy s'occupait, entre autres choses, des hôpitaux offerts par le Canada à la France.

47 Art. cit., 447.

48 Pierre Savard, «Les Canadiens-Français et la France de la 'Cession' à la 'Révolution tranquille'», in Le Canada et le Québec sur la scène internationale, 489.

49 A.P.C., Fonds Borden, Roy à Gouin, 9 janvier 1913. Le même jour, il démissionnait du conseil d'administration de la «caisse hypothécaire canadienne» comme Borden le lui avait demandé le 18 octobre 1912. La double démission avait été exigée par l'ordre en Conseil P.C. 3278 du 20 décembre 1912.

50 Id., Roy à Borden, 9 janvier 1913; Roy à Pelletier, 4 mars 1914.

51 Id., Roy à Pelletier, 29 septembre 1914.

52 A.P.C., R.G. 2, série I, ordre en Conseil P.C. 3536, 20 décembre 1912. 
C'étaient l'hôpital de Dinard, les hôpitaux militaires de SaintCloud et de Joinville-le-Pont, et l'hôpital de l'Université Laval, situé lui aussi à Saint-Cloud. Mais le statut du poste ne fut en rien modifié pendant la guerre. Au contraire, le haut commissariat de Londres renforça son contrôle sur le commissariat de Paris. Cela tient à la fréquence des visites des ministres canadiens dans la capitale britannique au cours de cette période. Lors de la Conférence de la paix, les Dominions furent représentés à Paris et leurs ministres plénipotentiaires signèrent les traités. Ils avaient ainsi obtenu, grâce à l'initiative de Robert Borden d'être considérés "comme distincts à l'intérieur du groupe impérial». De plus, ils furent admis à titre individuel au sein de la Société des Nations. Le Canada pouvait donc espérer obtenir, dans un proche avenir, une représentation diplomatique indépendante et désormais officiellement reconnue ${ }^{53}$.

\section{IV - LE RETOUR DES LIBÉRAUX ET LA LÉGATION}

\section{A - Les projets de Roy}

En janvier 1921, Roy exposa à Mackenzie King, alors chef de l'opposition, un plan de réorganisation du commissariat, qui lui semblait inadapté à l'importance nouvelle du Dominion ${ }^{54}$. Il soulignait que le commissariat de Paris pouvait conférer au Canada un prestige tout particulier. Il ne devait donc pas être traité sur le même pied que les autres agences. Roy insistait à plusieurs reprises sur la nécessité de renforcer l'image de marque de son pays en France, car les relations entre cette dernière et le Canada étaient appelées à se développer encore. "Il n'y a pas d'autres pays au monde, écrivait-il, qui aient d'aussi bonnes raisons d'être toujours en termes amicaux.» Le Canada avait éveillé la sympathie dans le coeur des Français grâce à l'héroïsme de ses soldats et à son grand effort de guerre. Le commissaire qualifiait d'ailleurs les Français de «peuple merveilleux, plein d'idéal et de générosité».

Après la victoire du parti libéral aux élections fédérales de décembre 1921, King, devenu premier ministre, s'était aussi attribué le portefeuille des Affaires extérieures. Roy lui écrivit alors pour appuyer encore sur la nécessité de développer la collabora-

53 Voir Claude Le Gris, L'entrée du Canada sur la scène internationale (1919-1927)

(Paris, P. U. F., 1966), 95 p.

54 A.P.C., Fonds King, Roy à King, 18 janvier 1921 . 
tion franco-canadienne. On semblait la rechercher plus directement désormais du côté du gouvernement français ${ }^{55}$. C'était la période euphorique des «missions» françaises au Canada ou canadiennes en France ${ }^{56}$. Pour maintenir la continuité de ces échanges, Roy travaillait à la fondation d'une Maison canadienne à la Cité universitaire de Paris. À ce sujet, le commissaire, qui ne représentait plus le Québec depuis janvier 1913, demandait la permission de rentrer au Canada pour assister à la session de la législature de Québec ${ }^{57}$. Il avait en effet «des questions importantes à soumettre au Gouvernement et au Parlement de la Province de Québec, en ce qui concerne l'organisation définitive d'une Maison Canadienne à Paris». Comme s'il était gêné de paraître représenter indûment la province de Québec, Philippe Roy se justifiait en poursuivant:
Remarquez, mon cher Premier Ministre, que ce projet d'une Maison Canadienne à Paris pour nos Etudiants, n'a pas exclusivement un caractère et une portée provinciale. Je ne connais rien qui pourrait avoir des effets plus généreux au point de vue des relations futures entre la France et le Canada qu'une institution ayant le caractère de celle que nous voulons former en France. ${ }^{58}$

En 1923, Roy échafauda des plans grandioses pour améliorer la situation matérielle du commissariat. Le 3 février 1923, sir Henry Thornton, devenu président du Canadien National Railways, lui demandait de trouver un local adapté à l'importance du C.N.R. à Paris, en dehors des services purement commerciaux ${ }^{59}$. Il suggérait l'acquisition d'un immeuble situé de préférence boulevard des Capucines, pas très loin de l'intersection de celui-ci avec la rue Edouard VII. Le rez-de-chaussée serait occupé par le C.N.R. et les étages supérieurs réservés aux bureaux du «Haut Commissaire ${ }^{60}$. Roy répondait le 9 mars suivant en approuvant totalement les vues du président du C.N.R. et en les amplifiant

55 Id., le 13 décembre 1921. Roy écrivait: «Les relations franco-canadiennes peuvent être améliorés au point de vue économique. Le Gouvernement français est extrêmement anxieux de faire un nouveau Traité Commercial avec le Canada. J'ai eu, depuis six mois, des entrevues intéressantes avec les Directeurs du Ministère des Affaires Étrangères et du Ministère du Commerce.»

56 Mission du général Pau en 1919, du maréchal Fayolle en 1921 avec le trainexposition français à travers le Canada; mission canadienne en 1923.

57 A. P. C., Fonds King, Roy à King, 16 janvier 1922.

58 Id., 16 janvier 1922 .

59 Id., Thornton à Roy, 3 février 1923.

60 Roy n'était que commissaire général. 
encore ${ }^{61}$. En fait, ce projet ambitieux échoua devant le coût élevé de l'hôtel Scribe ${ }^{62}$. En devenant haut-commissaire, Roy ne voyaitil pas le moyen de secouer la tutelle du haut-commissaire à Londres et même celle de l'ambassadeur de Grande-Bretagne à Paris?

\section{B - La légation canadienne à Paris}

Roy se préoccupait naturellement beaucoup du statut du représentant canadien en France. N'était-ce pas enfin le moment favorable pour demander au Gouvernement de la République la reconnaissance officielle sans opposition britannique? C'est ce qu'il suggérait à King en avril 1925:

On ne comprend plus ici, surtout depuis la guerre, que le représentant du Canada à Paris n'ait pas un rôle officiel, au moins égal à celui des représentants de Cuba ou du Libéria (...)

Il est aussi regrettable, à mon avis, que le représentant du Canada à Paris soit exclu de la liste des invitations officielíes, soit chez le Président de la République, soit chez les Présidents des Chambres du Parlement, parce qu'il y a une Ambassade Anglaise à Paris. ${ }^{63}$

Le commissaire ajoutait que les Français ${ }^{64}$ et les Canadiens n'admettaient pas cette exclusion et cette humiliation. Cette situation créait une certaine tension entre le commissariat canadien et l'ambassade britannique ${ }^{65}$. Le gouvernement français était maintenant prêt à reconnaître une position officielle au commissariat pour être plus à l'aise avec l'ambassade d'Angleterre. Roy suggérait d'essayer d'obtenir d'abord la réciprocité complète avec le consulat général de France à Montréal. Le consul de France jouis-

61 A.P.C., Fonds King, Roy à Thornton, 9 mars 1923. On placerait dans le sous-sol une salle de cinéma pour la propagande commerciale et touristique. Le rez-de-chaussée serait consacré à une exposition permanente de produits canadiens; il abriterait aussi les bureaux d'immigration et ceux du C.N.R. Les bureaux du «Haut Commissaire» et du commissaire au commerce seraient situés au premier étage. L'espace demeuré libre serait loué. Roy conseillait l'achat de l'hôtel Scribe. Son prix allait effrayer le ministère des Finances et le Parlement, mais le gouvernement s'apprêtait à acheter fort cher un immeuble pour reloger le haut-commissariat de Londres. Il ne pouvait pas faire moins pour Paris.

62 Id., Roy à King, 11 avril 1925 .

63 Id., 11 avril 1925.

64 Par exemple les membres du Comité France-Amérique, présidé par G. Hanotaux.

65 Roy était exclu des déjeuners officiels à l'ambassade. Lord Crewe, l'ambassadeur du Royaume-Uni, «se rendait compte, depuis longtemps, que le Canada ne se servait guère de l'ambassade dans ses relations avec la République française et que cette situation était anormale». Dandurand, op. cit., 312. 
sait en effet de la franchise douanière et de l'exemption des impôts fédéraux et provinciaux.

Grâce à l'intervention du Baron de Vitrolles, au moment où il a été nommé Consul Général au Canada ${ }^{66}$, j'ai obtenu la franchise douanière, à titre de réciprocité. Je suis sûr que si votre gouvernement exprimait le même désir au Consul de France à Montréal, en ce qui regarde les impôts, et toujours à titre de réciprocité, nous obtiendrions cette exemption immédiatement. ${ }^{67}$

Mais le principal responsable de la transformation du commissariat en légation fut le sénateur libéral et francophile Raoul Dandurand ${ }^{68}$. Il signalait la nécessité de cette nouvelle étape dans les relations franco-canadiennes à son ami Gabriel Hanotaux, par une lettre du 12 février 1925, à laquelle le président du Comité France-Amérique répondait:

Oui, vous devriez avoir un ministre à Paris. J'insiste de toutes mes forces pour cette solution qui est la vraie. Vous seul pouvez mener la chose à bonne fin. M. Roy est si populaire ici. La situation est des plus favorables. ${ }^{6}$.

À ces bonnes dispositions du milieu politique français correspondait l'évolution générale du Commonwealth britannique. Du 19 octobre au 23 novembre 1926 se tint à Londres la célèbre conférence impériale qui reconnut pratiquement l'indépendance des Dominions. King, accompagné de Lapointe, ministre de la Justice, et de Taschereau, premier ministre du Québec, y représentait le Canada. Il se rendit ensuite à Paris pour rencontrer Philippe Roy et aborder le problème de la légation. Le sénateur Dandurand avait déjà préparé le terrain auprès du premier ministre. Il écrivait à Roy avant la conférence:

Je vais tâcher d'aborder la question concernant votre Commissariat, tant au point de vue matériel qu'au point de vue politique et administratif avec M. King, demain ou samedi, nous ferons route ensemble d'Ottawa à Québec - afin de le préparer pour la conversation que vous aurez avec lui à Londres ou à Paris. ${ }^{70}$

66 En 1924.

67 A.P.C., Fonds King, Roy à King, 11 avril 1925.

68 R. Dandurand, Mémoires, chap. XXIII, L'établissement de légations à Paris et à Tokio, 309-315, Dandurand fut ministre d'État de 1921 à 1930 et président de l'assemblée de la Société des Nations en 1925.

69 A.P.C., Fonds Raoul Dandurand, M. G. 27, III B 3, Hanotaux à Dandurand, 14 mars 1925 .

70 Id., Dandurand à Roy, 6 octobre 1926. 
À la fin de novembre 1926, King rencontra donc Roy à Paris. Les deux hommes évoquèrent la création de la légation canadienne de Washington où un ministre plénipotentiaire venait d'être nommé ${ }^{71}$. Il fut aussi question d'un "changement similaire» à réaliser en France rapidement ${ }^{72}$. King voulait en effet établir la légation de Paris dès que celle de Washington aurait commencé à fonctionner ${ }^{73}$. Peu après cette entrevue, dans une lettre du 3 décembre 1926 à King, Roy revenait sur cette affaire de légation en insistant sur les bonnes dispositions des autorités françaises:

Nous avons eu une agence en France depuis près de cinquante ans. Elle a atteint, au moins en pratique, l'importance d'une légation. Le gouvernement français, je le sais, accueillerait favorablement un changement de notre statut, et nous accorderait immédiatement la réciprocité en nommant un ministre à Ottawa. ${ }^{74}$

Roy précisait que ce nouveau statut n'affecterait pas ses relations avec l'ambassade britannique à Paris, avec laquelle il entretenait maintenant les meilieurs rappoits ${ }^{75}$.

Dès le 5 janvier 1927, Mackenzie King télégraphiait à Philippe Roy qu'il avait discuté de la situation de l'agence de Paris avec le sénateur Dandurand. Roy devait avertir le ministre français des Affaires étrangères, Aristide Briand, que le gouvernement canadien souhaitait la publication du communiqué annonçant la transformation du commissariat en légation, à la date qui conviendrait au gouvernement français ${ }^{76}$. De ce côté, l'idée du développement des relations avec le Canada continuait à faire son chemin ${ }^{77}$.

71 Vincent Massey, le 5 novembre 1926. Voir Le Gris, op. cit., 79.

72 A.P.C., Fonds King, Roy à King, 3 décembre 1926.

73 Massey présenta ses lettres de créance au président Coolidge le 8 février 1927, Le Gris, op. cit., 80.

74 A.P.C., id.

75 Il lui faudrait néanmoins attendre l'automne 1927 pour être «officiellement invité à déjeuner chez lord Crewe». Dandurand, op. cit., 312.

76 A. P. C., id., King à Roy, 5 janvier 1927.

77 Le 27 août 1927, un sénateur français envoyé en mission aux États-Unis et au Canada faisait rapport à Briand sur son voyage et déclarait, à propos de la représentation française au Canada:

Reste la question posée depuis un certain temps de savoir s'il ne faudrait pas nommer au Canada un Ministre au lieu d'un Consul Général. Il me paraît que notre situation et notre influence comporteraient une fonction de ce genre dans ce grand pays en plein développement de commerce et de population. Ce serait une aide apportée à l'élément français qui se maintient et que la province de Québec n'abandonnera jamais, mais qui actuellement se trouve au Canada devant une invasion américaine qui va s'accroissant.

A.P.C., Fonds Dandurand, correspondance avec Briand, 27 août 1927. Le rapport est incomplet; il manque en particulier la dernière page avec le nom du sénateur. 
En novembre 1927, à la demande de King, Dandurand rencontra Briand à Paris pour lui proposer, au nom de son gouvernement, l'ouverture de la légation canadienne ${ }^{78}$. Tenu au courant de la négociation par le sénateur, Roy se réjouit de cette décision. Elle représentait, disait-il, un grand pas en avant pour le «canadianisme». Roy était d'autant plus satisfait que Dandurand l'avait prévenu qu'il serait nommé ministre plénipotentiaire et placé à la tête de la nouvelle légation ${ }^{79}$. C'est ainsi que le 10 janvier 1928 avait lieu la création simultanée d'une légation française à Ottawa et d'une légation canadienne à Paris. Le 12 septembre, Jean Knight était nommé ministre plénipotentiaire de France au Canada; et Philippe Roy recevait sa nomination officielle à Paris pour le ler octobre, après avoir présenté ses lettres de créance au président Doumergue le 29 septembre à Rambouillet.

\section{CONCLUSION}

Il faut tout d'abord signaler que la province de Québec a joué un rôle de moteur dans les années 1880 . Elle fut responsable de la création de l'agence générale et lui a fourni ses titulaires ${ }^{80}$. Ses premiers ministres ont franchi plusieurs fois l'Atlantique pour faire des visites officielles en France: Chapleau en 1881, Mercier en 1888 et en 1891; de nouveau Chapleau, comme lieutenant-gouverneur du Québec, en $1893^{81}$. Mais ensuite le Québec, ébloui par Laurier, laisse la première place à Ottawa.

Quant à elle, la politique du gouvernement fédéral présente quatre phases successives qui correspondent aux changements parlementaires à Ottawa. Le gouvernement conservateur a mis le train

78 Dans ses Mémoires, 310-312, Dandurand précise qu'en 1927 le Canada avait décidé de restreindre l'immigration japonaise. Ottawa désirait discuter les termes de cette restriction avec le gouvernement nippon sans passer par Londres. Il fallait donc établir une légation canadienne à Tokio et une légation japonaise à Ottawa. Le cabinet libéral, "pour des raisons sentimentales» décida "de se tourner vers la France pour l'inviter la première, après les États-Unis, à établir une légation à Ottawa». C'est alors que King chargea Dandurand de négocier avec Briand après avoir averti le secrétaire au Foreign Office, sir Austen Chamberlain, et l'ambassadeur britannique à Paris.

79 A.P.C., id., Roy à King, 24 décembre 1927. Pourtant, les dirigeants du Comité France-Amérique croyaient savoir que Roy avait obtenu sa retraite; Gabriel-Louis Jaray, directeur général du Comité, proposait confidentiellement à Dandurand le nom d'Edouard Montpetit comme successeur de Roy, A.P.C., Fonds Dandurand, Jaray à Dandurand, 9 octobre 1928. cois.

80 Il est désormais entendu que le représentant du Canada en France soit un Québé-

81 C'est aussi le cas du sous-ministre de la Colonisation du Québec, le curé Labelle, en 1885 et en 1890 . 
en marche en 1882. Pour lui, l'agence de Paris était essentiellement commerciale et financière. Il la plaçait sous le contrôle étroit du haut-commissariat de Londres. Sa fondation ressemblait plus à un expédient de politique intérieure qu'à une vue à long terme du développement des relations franco-canadiennes. Puis le gouvernement libéral de Laurier encouragea l'immigration française au Canada, en réactivant les services du commissariat parisien. Pour servir sa politique "nationale», il songea à transformer progressivement les agences canadiennes en consulats. Mais avec le retour au pouvoir des conservateurs en 1911, l'agence de Paris fut à nouveau limitée à son rôle commercial et financier. Après la Conférence de la paix puis la rentrée des libéraux en 1921, le gouvernement canadien s'achemina résolument vers la transformation du commissariat en légation. Il bénéficiait dès lors de l'appui assuré du gouvernement français.

En effet, la politique française à l'égard de l'agence canadienne reflète deux attitudes différentes. Avant la première guerre mondiale, la France se montra très prudente dans ses reiations avec le Canada. Elle freina la marche du Dominion vers l'indépendance en soulignant sa place de "colonie» britannique. Mais à partir de 1919, la France reconnut progressivement le droit du Canada à la personnalité internationale. Il faut en chercher l'explication dans la politique française à l'égard de l'Angleterre. Du temps des rivalités coloniales franco-britanniques en Afrique et en Asie, la France ne tenait pas à susciter de nouveaux motifs de querelle à propos de l'Amérique du Nord. Puis la politique d'entente cordiale conduisit la France à ne pas froisser la susceptibilité impériale de son partenaire. Après la brillante participation canadienne à la grande guerre, la France, qui se heurtait souvent à la GrandeBretagne dans sa politique d'application du traité de Versailles, eut moins de raisons de ménager le Royaume-Uni. Celui-ci de son côté se résignait à reconnaître l'indépendance des Dominions. La France ne fit en l'occurrence que s'adapter à la politique d'émancipation voulue par le Canada. La présidence de l'Assemblée générale de la Société des Nations n'avait-elle pas été attribuée au Canadien Raoul Dandurand dès 1925, avec l'appui de la France. 